\title{
Dry Eye Syndrome: A Multifactorial Disease
}

Kottaiyan R

Senior Associate, The Ocular Surface Laboratory Flaum Eye Institute, University of Rochester Medical Center G-3122, 601 Elmwood Avenue, Box 314, Rochester, NY 14642, USA.

\section{*Corresponding Author:}

Ranjini Kottaiyan, B. S Opt (OD), MBA ,

Senior Associate, The Ocular Surface Laboratory Flaum Eye Institute, University of Rochester Medical Center G-3122, 601 Elmwood Avenue, Box 314, Rochester, NY 14642, USA.

Tel: +1-585-273-4874

Fax: +1-585-276-2432

E-mail: ranjini_kottaiyan@urmc.rochester.edu

Received: November 12, 2014

Published: November 21, 2014

Citation: Kottaiyan R (2014) Dry Eye Syndrome: A Multifactorial Disease. Int J Ophthalmol Eye Res. 02(4e), 1. doi: http://dx.doi. org/10.19070/2332-290X-140005e

Copyright: Kottaiyan $\mathbf{R}^{\circ}$ 2014. This is an open-access article distributed under the terms of the Creative Commons Attribution License, which permits unrestricted use, distribution and reproduction in any medium, provided the original author and source are credited.

Dry eye syndrome, a multifactorial disease of the ocular surface, is the leading cause for ophthalmology office visits, affecting around 60 million people in the United States alone. Due to a variety of factors, it is challenging to make a definitive diagnosis in the early stages of the disease. The ocular examination, including the fluorescein break up time and Schirmer's test has been a critical part of the diagnosis algorithm. But these tests are severely limited owing to their relative invasiveness and lack of specificity. To overcome the limitations of the current gold standards, there is a growing need for the use of noninvasive tear imaging technologies like the thermal imaging.

Thermography has been commercialized in the past 50 years and has a variety of clinical applications. It was first used to study ocular surface temperature changes by Mapstone in 1970. Ever since, there has been a number of research groups studying the application of Thermography as a diagnostic tool for dry eye disease. Current research indicates that the ocular surface temperature cooling rate of a dry eye is faster than that of a non-dry eye, because it has higher tear film evaporation. By observing the ocular surface temperature change, we can indirectly evaluate the tear film stability in both normal and dry eye subjects.

This capability provides a powerful tool to enhance our understanding of tear dynamics in space and time. It is important to note that investigating interactions between tear parameters is critical for an accurate understanding of how each tear parameter affects the others. To achieve this goal, it is important to combine tear imaging systems to measure the tear parameters simultaneously, as the tear system is highly dynamic.

However, there is an increased cost associated with commercializing the thermal imaging technique. Compared to the conventional dry eye tests like the Schirmer test and staining scores, a thermal camera is expensive. Also, current clinical tests can be interpreted instantaneously and easy to train and use. Thermal camera requires an advanced custom algorithm, and involves time and effort in making a diagnosis.

Increased efforts directed at devising a user friendly software, can result in the commercialization of the thermal camera as a quick and effective diagnostic tool in ophthalmology doctor offices, and has the potential to replace the current gold standards in dry eye diagnosis. 